\title{
Influence of temperature on the phase behaviors and techniques toward formation of palm oil esters nanoemulsion.
}

\begin{abstract}
Palm oil esters (POEs), which are newly synthetic liquid wax esters with HLB value of 9.34, were proposed as a lipophilic phase for formation of nanoemulsion. Phase diagrams show domination of two-phase regions at all emulsification temperatures ranging from 30 to $80 \mathrm{C}$. Spontaneous and temperature-induced emulsification, high-shear and high pressure homogenization were utilized to form nanoemulsion. However, only high pressure homogenization successfully produced droplets sizes in the nano range. Thus, it was used to optimize the stability properties of POEs nanoemulsion. The manipulation of processing temperatures during the formation of emulsions could be used in lowering the droplets size of the emulsion.
\end{abstract}

Keyword: Nanoemulsion; Palm oil esters; Phase behavior; Temperature. 